\title{
Meiotic behaviour of individual chromosomes in allotriploid Alstroemeria hybrids
}

\author{
SA Kamstra ${ }^{1}$, JH de Jong ${ }^{2}$, E Jacobsen ${ }^{1}$, MS Ramanna ${ }^{1}$ and AGJ Kuipers ${ }^{1}$ \\ ${ }^{1}$ Laboratory of Plant Breeding, The Graduate School of Experimental Plant Sciences, Wageningen University, PO Box 386,6700 AJ \\ Wageningen, The Netherlands; ${ }^{2}$ Laboratory of Genetics, The Graduate School of Experimental Plant Sciences, Wageningen University, \\ Arboretumlaan 4, 6703 BD Wageningen, The Netherlands
}

Chromosome association and chiasma formation were studied in pollen mother cells at metaphase I of four allotriplod $\mathrm{BC} 1$ plants $(2 n=3 x=24)$ obtained from the backcross of the hybrid Alstroemeria aurea $\times A$. inodora with its parent $A$. inodora. We distinguished the chromosomes of both parental species by genomic in situ hybridization (GISH), whereas the individual chromosomes were identified on the basis of their multicolour FISH banding patterns obtained after a second hybridization with two species-specific satellite repeats as probes. All the four $\mathrm{BC} 1$ plants possessed two genomes of $A$. inodora and one of $A$. aurea. Variable numbers of recombinant chromosomes, resulting from meiotic recombination in the interspecific hybrid, were present in these plants. The homologous $A$. inodora chromosomes generally formed bivalents, leaving the homoeologous $A$. aurea chromosomes unassociated. High frequencies of trivalents were observed for the chromosome sets that contained recombinant chromosomes, even when the recombinant segments were small. Chromosome associations in the trivalents were restricted to homologous segments. The implications of the absence of homoeologous chromosome pairing on gamete constitution and prospects for introgression in Alstroemeria are discussed.

Heredity (2004) 93, 15-21, advance online publication, 21 April 2004; doi:10.1038/sj.hdy.6800465

Keywords: Alstroemeria aurea; $A$. inodora; fluorescent in situ hybridization; karyotype; meiosis; translocation

\section{Introduction}

Allotriploid (AAB) plants contain two genomes of one species and one genome from a different species. Generally, they are obtained from crossing an allotetraploid (eg AABB) with one of the diploid parents (eg AA). Such backcross derivatives, also referred to as sesquidiploids, occasionally produce balanced haploid $(n=x)$, diploid $(n=2 x)$ and triploid $(n=3 x)$ gametes, as well as unbalanced gametes with an aneuploid number of chromosomes. The unbalanced gametes of allotriploids have been used to produce monosomic additions in several crops, such as Nicotiana (Suen et al, 1997), Brassica (Chen et al, 1997), Beta (Mesbah et al, 1996), Allium (Peffley et al, 1985, Shigyo et al, 1996) and Lolium (Morgan, 1991).

Alstroemeria (family Alstroemeriaceae, order Liliales) is a relatively young ornamental crop. Most cultivars have originated through hybridization of diploid species $(2 n=2 x=16)$ that are endemic to Chile and Brazil (Goemans, 1962). Interspecific hybrids, including interChilean species hybrids and hybrids between Chilean and Brazilian species, are often highly sterile but unreduced $(2 n)$ gametes occur in variable frequencies and formed the basis of the present-day triploid $(2 n=3 x=24)$ and tetraploid $(2 n=4 x=32)$ cultivars (Ramanna, 1992). To evaluate the breeding prospects

Correspondence: AGJ Kuipers, Binnenhaven 5, 6709 PD Wageningen, The Netherlands. E-mail: anja.kuipers@wur.nl

Received 19 August 2002; accepted 7 October 2003; published online 21 April 2004 for introgression of desired traits from $A$. aurea into A. inodora, Kamstra et al (1999a, b) backcrossed the interspecific diploid $(2 n=2 x=16)$ hybrid of $A$. aurea $\times$ $A$. inodora with $A$. inodora. In the $\mathrm{BC} 1$ progeny, aneuploids as well as allotriploids were observed. The allotriploids resulted from unreduced (2n) gametes produced by the F1 hybrid and, consequently, contained two genomes of $A$. inodora $(\mathrm{P})$ and one of $A$. aurea (A) (Kamstra et al, 1999b). Identification of all chromosomes in the BC1 plants was achieved on the basis of genomic and fluorescent in situ hybridization (GISH and FISH). GISH, which enabled differentiation between the $A$. inodora and $A$. aurea chromatin, revealed the existence of four to 10 recombinant chromosomes in the different BC1 plants (Kamstra et al, 1999a, b). The number, size and position of the exchanged segments in these chromosomes varied between plants. In addition, structurally altered chromosomes were detected in some of the BC1 plants (Kamstra et al, 1999b). These chromosomes contained hybridization sites for species-specific probes at unexpected locations, not found in parental species (Kamstra et al, 1997), indicating the presence of chromosomal rearrangements between the $A$. aurea and $A$. inodora genome (Kamstra et al, 1999b).

This paper describes the meiotic analysis of four BC1 plants using FISH and GISH sequentially applied to spread pollen mother cells at metaphase I to telophase II. To use the allotriploids for further backcrossing, it was essential to analyse meiosis in order to determine the types of gametes that are produced and to predict the consequences of observed meiotic disturbances for further backcrossing. The aims of this investigation were: 
(i) to study the meiotic associations of each individual chromosome in the allotriploids and to estimate pairing frequencies; (ii) to confirm the previously hypothesized structural rearrangements between the chromosomes of both parental species on the basis of metaphase I configurations of the structurally altered chromosomes; (iii) to analyse the behaviour of individual genomes and chromosomes, especially the $A$. aurea chromosomes, during different meiotic stages.

\section{Materials and methods}

\section{Plant material}

Four allotriploid plants BC1-1 (95SK04), BC1-2 (95SK17), BC1-3 (96SK402) and BC1-4 (96SK403) were derived from the backcross between the hybrid $A$. aurea $\times A$. inodora (AP) and its parent $A$. inodora (P) (Kamstra et al, 1999a). A combination of GISH for genome identification and FISH for individual chromosome identification was used to assess the karyotypes of these allotriploids (PPA) with two $A$. inodora $(\mathrm{P})$ genomes and one $A$. aurea (A) genome (Kamstra et al, 1999b; Figure 1). Chromosomes were classified according to Kamstra et al (1999b).

\section{Meiotic preparations of the $\mathrm{BC} 1$ plants}

Anthers were fixed in 3:1 ethanol:acetic acid for approximately $1 \mathrm{~h}$ and stored in $70 \%$ ethanol at $-20^{\circ} \mathrm{C}$ until use. Before cell spreading, the material was digested with a mixture of pectolytic enzymes $(0.4 \%$ pectolyase $\mathrm{Y} 23,0.4 \%$ cellulase RS and $0.4 \%$ cytohelicase in $0.1 \mathrm{M}$ citrate buffer, $\mathrm{pH} 4.5$ ) for $2-3 \mathrm{~h}$ at $37^{\circ} \mathrm{C}$. The softened anthers were squashed in fresh $45 \%$ acetic acid. Preparations containing pollen mother cells from metaphase I to anaphase II were selected. Sequential in situ hybridization experiments (GISH and FISH with two species-specific probes) were performed on most slides.

\section{Genomic in situ hybridization (GISH)}

Probe preparation and GISH were performed as previously described (Kuipers et al, 1997; Kamstra et al, 1999b). In short, two differentially labelled genomic DNA probes of the parental species A. aurea (biotinlabelled) and $A$. inodora (digoxigenin-labelled) were preannealed for $2 \mathrm{~h}$ at $58^{\circ} \mathrm{C}$, hybridized overnight to denatured slides, and detected using Cy3- (Jackson ImmunoResearch Laboratories) and FITC/Alexa 488conjugated antibodies (Molecular Probes Inc.).

\section{Fluorescent in situ hybridization (FISH)}

The repeats D32-13 (specific for $A$. inodora; EMBL accession number AJ228814) and A001-I (specific for $A$. aurea; EMBL accession number Y10977) were labelled by PCR with biotin-16-dUTP or digoxigenin-11-dUTP, respectively, and hybridized to the meiotic cells as described in Kamstra et al (1997, 1999a, b). The biotinand digoxigenin- labelled probes were detected using Cy3- (Jackson ImmunoResearch Laboratories) and FITC/ Alexa 488-conjugated antibodies (Molecular Probes Inc.).

\section{Results}

All the four allotriploids BC1-1, BC1-2, BC1-3 and BC1-4 $(2 n=3 x=24)$ possess two genomes of $A$. inodora and one of $A$. aurea. These plants contain several recombinant chromosomes, which are described in a previous paper (Kamstra et al, 1999b). In total, chromosome association frequencies were analysed in 542 pollen mother cells (PMCs) at metaphase $\mathrm{I}$ in the four allotriploids to establish the pairing frequencies. Pairing was studied per set of three homo(eo)logous chromosomes. This was analysed for all eight sets of homoeologous chromosomes in all plants. In addition, chromosome behaviour at later meiotic stages was established.

Metaphase I chromosome associations in the $\mathrm{BC} 1$ plants We first analysed the number of univalents (I), bivalents (II) and trivalents (III) in PMCs at metaphase I of the four allotriploids BC1-1, BC1-2, BC1-3 and BC1-4. Figure 2 shows examples obtained from BC1-3 that are representative for all the four plants. Unequivocal identification of individual chromosomes was based on sequential GISH and FISH patterns (Figure $2 \mathrm{a}, \mathrm{b}$ ). In all plants, the three homo(eo)logous chromosomes of each set either formed one bivalent + one univalent or one trivalent. The trivalents were observed for all eight chromosome sets per plant in variable frequencies, ranging from 2.0 to $100 \%$ (Table 1). Bivalent associations were mainly confined to the two homologous $A$. inodora chromosomes, whereas their $A$. aurea homoeologue was present as a univalent (eg Figure 2b). Three univalents were not observed for any of these chromosome sets.

Sets of three homo(eo)logous chromosomes, containing one or two recombinant chromosomes (recombinant sets), predominantly formed trivalents (Figure 2a). The frequency of trivalents in recombinant sets ranged from $40.3 \%$ for the chromosomes 6 in BC1-3 to $100 \%$ for both recombinant sets (1 and 5) in BC1-2 (Table 1), which is significantly higher than for non-recombinant sets (twosample one-tailed $t$-test, assuming equal variances; $P=1.6 \times 10^{-15}$ ).

For all recombinant sets, GISH clearly revealed that the chromosome associations in the trivalents were restricted to homologous segments (Figure 2a, c), that is, no associations between homoeologous parts were detected. In general, the $A$. aurea segments in these trivalents were unpaired (Figure 2a), except for the recombinant sets in which two homologous $A$. aurea segments were present such as set 8 of BC1-1 or set 4 of BC1-3 (Figure 2a, trivalent of $4 \mathrm{~A} / 5 \mathrm{P}+5 \mathrm{P}+5 \mathrm{P} / 4 \mathrm{~A})$.

In the case of nonrecombinant sets, we observed far more bivalent + univalent configurations than trivalent configurations, with the two $A$. inodora chromosomes forming the bivalent and the $A$. aurea chromosome the univalent (Figure 2a, b). The frequency of bivalent + univalent ranged from 42.1 to $97 \%$ (Table 1). The occurrence of trivalents in the nonrecombinant sets, which ranged from 3 to $57.9 \%$ per set (Table 1), indicated pairing and recombination between the homoeologous $A$. inodora and $A$. aurea chromosomes (see Figure $2 \mathrm{a}, \mathrm{b}$ : trivalent of 1P-1P-1A). Compared to all other nonrecombinant sets of chromosomes, the sets of chromosomes 1 of BC1-4 and BC1-3 formed significantly $\left(\chi^{2} ; P<0.05\right)$ more trivalents (Table 1 ).

\section{Metaphase 1 configurations of the structurally altered} chromosomes

In the BC1-3 a pseudo-iso-chromosome $2 \mathrm{~A} / 2 \mathrm{P}$ was present, containing two homologous segments of the 
a

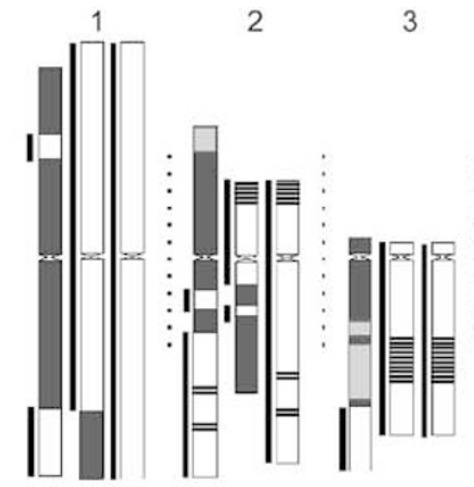

BC1-1 1A/1P 1P/1A 1P 2A/2P 2P/2A 2P 3A/4P 4P 4P

4

5

6

7

8

b

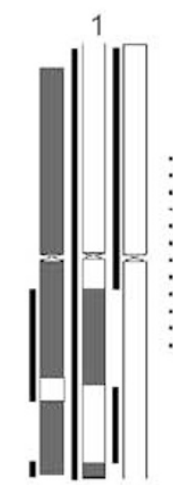

2

3

4A 5P 5P 5A/3P 3P/5A 3P

6A 6P 6P

4

5

5

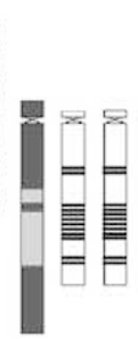

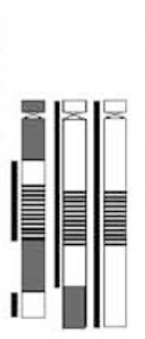

4 A $5 P$ 5P $5 A / 3 P$ PP/5A 3P
6

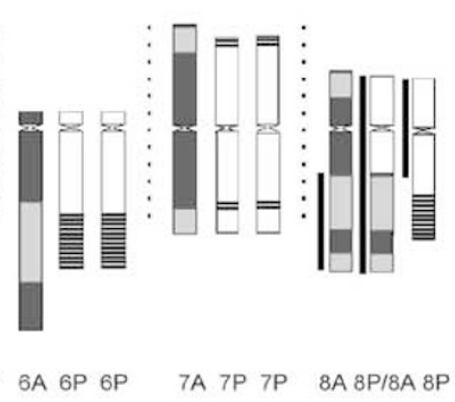

7

8

BC1-2 1A/1P 1P/1A 1P

2A $2 P 2 P$

$3 A 4 P 4 P$

2

3

4

5

6

7A 7P 7P

8A 8 P 8 P

C
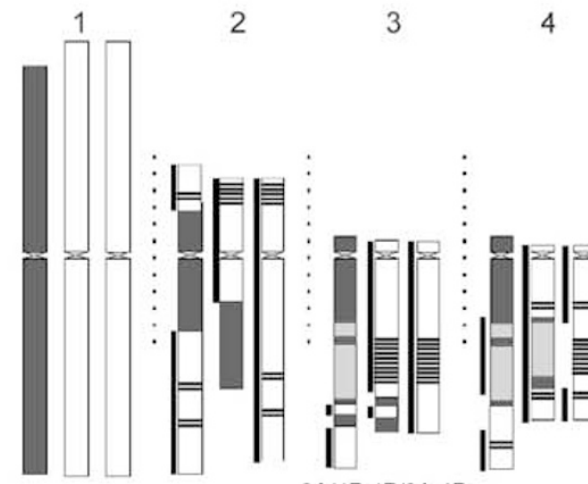

BC1-3 1A $1 \mathrm{P}$ 1P 2A/2P 2P/2A $2 \mathrm{P}$

$3 A / 4 P$ 4P/3A 4P 4 A/5P 5P/4A 5P
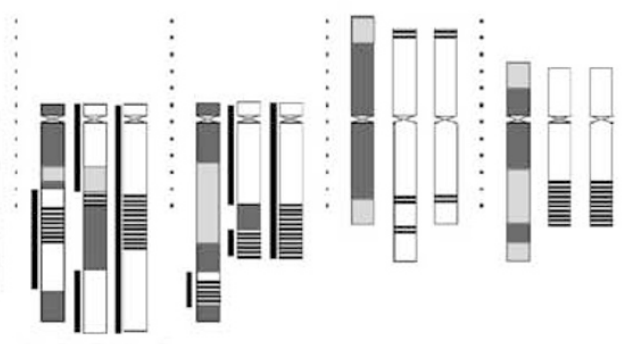

d

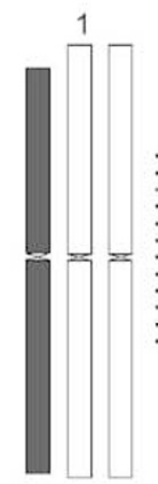

2

3

4

A/3P 3P/5A 3P

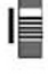

6A/6P 6P/6A GP

7A 7P 7P

8 A $8 \mathrm{P} 8 \mathrm{P}$

d

3

6

7

8

BC1-4

1A 1P 1P

2A 2 P 2 P

3A 4P 4P
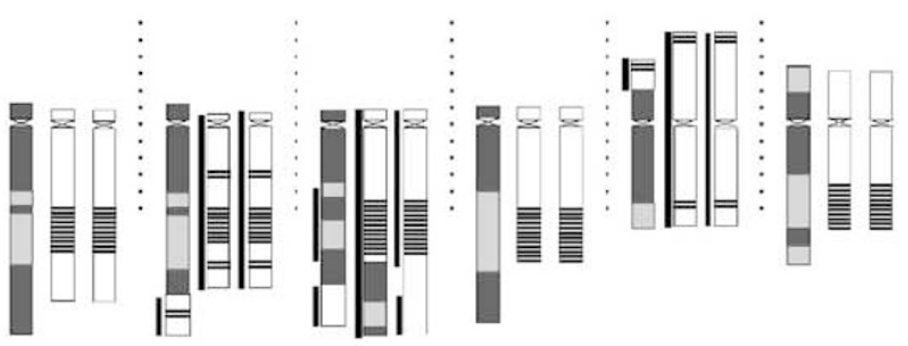

Figure 1 Karyotypes of the four allotriploids. (a) $\mathrm{BC}_{1}-1,(\mathbf{b})$. $\mathrm{BC}_{1}-2,(\mathbf{c}) \mathrm{BC}_{1}-3,(\mathrm{~d}) \mathrm{BC}_{1}-4$. A. aurea and A. inodora chromatin is indicated in dark grey and white, respectively. All plants contain recombinant chromosomes. The bars next to the chromosomes of recombinant sets indicate that for these chromosome segments homologous segments are present. Light grey boxes: A001-I hybridization sites on A. aurea chromosomes. Lined boxes: D32-13 hybridization sites on A. inodora chromosomes. 
short arm of $2 \mathrm{P}$ distally on both arms. This recombinant chromosome was considered to be the result of two crossovers between assumed translocated homoeologous segments in the parental hybrid (Kamstra et al, 1999b; Figure 2d4). To confirm the nature of the pseudoiso-chromosome, we analysed chromosome configura-

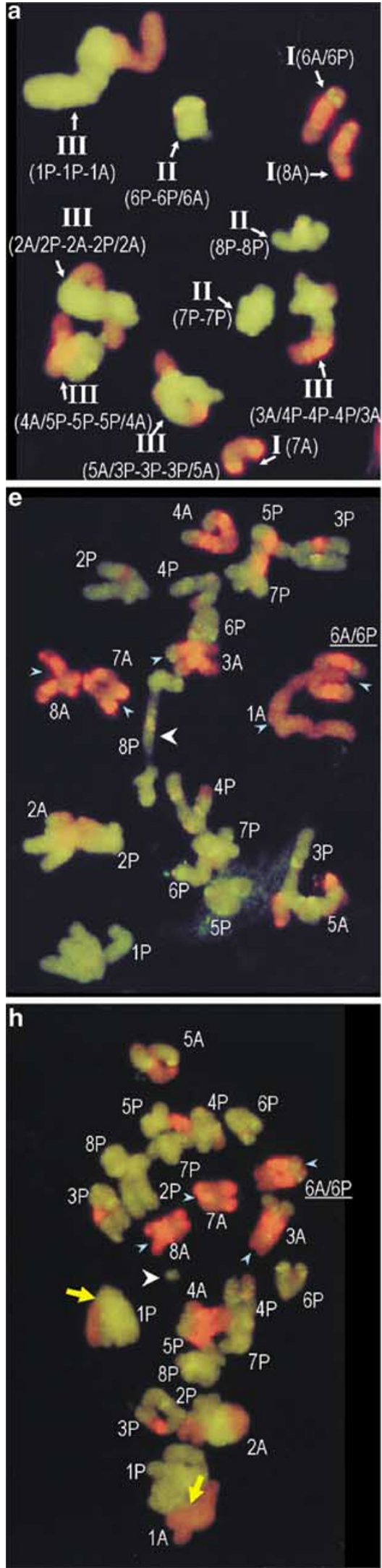

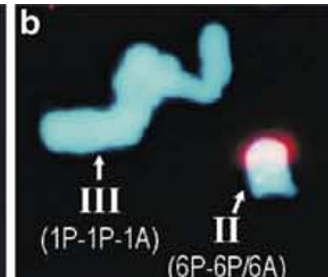

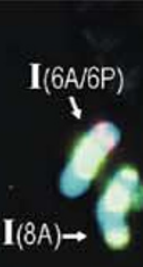

III

$(2 A / 2 P-2 A-2 P / 2 A)$

$$
\text { II }
$$

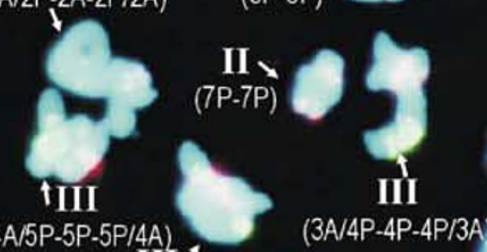

(4A/5P-5P-5P/4A)

III
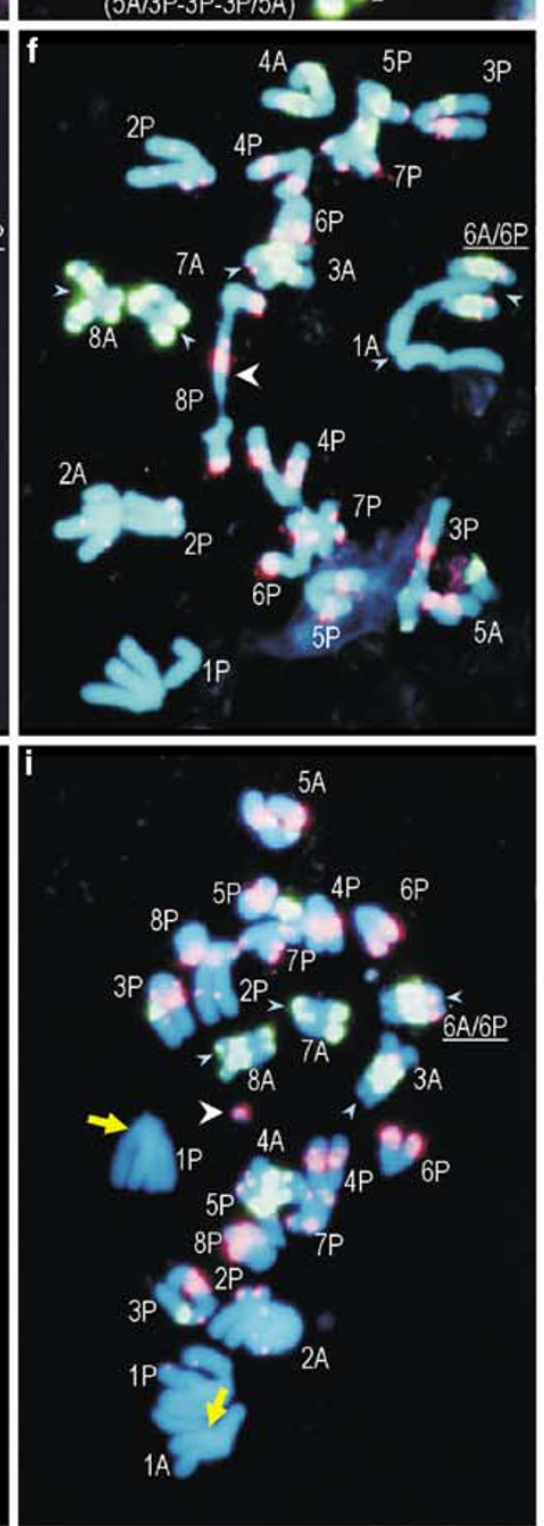
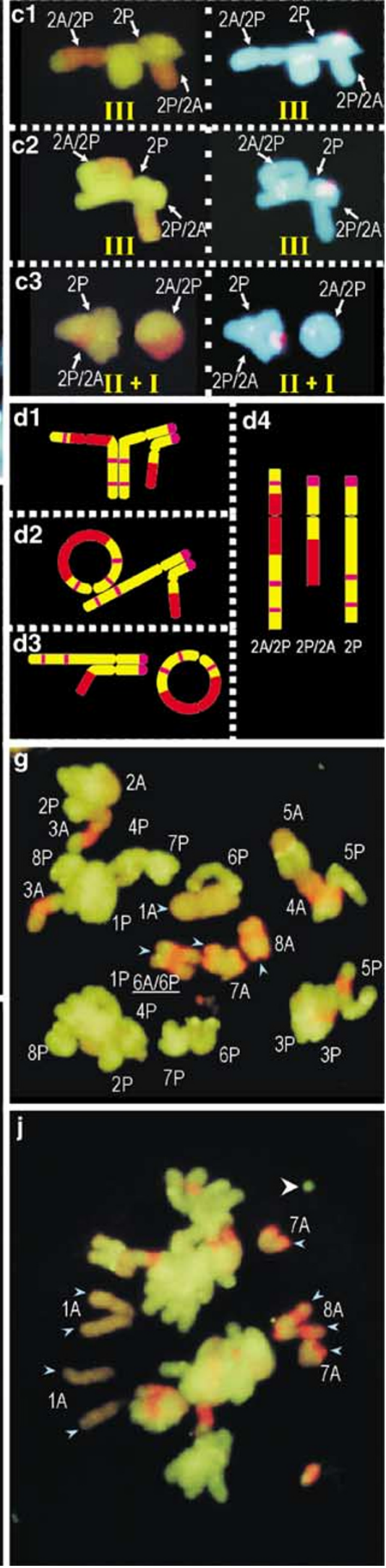
Table 1 Observed frequency (\%) of metaphase I associations (trivalent (III) or bivalent + univalent (II+I)) per nonrecombinant or recombinant set of three homo(eo)logous chromosomes

\begin{tabular}{|c|c|c|c|c|c|c|c|c|c|c|}
\hline \multirow[t]{2}{*}{ Genotype } & \multirow[t]{2}{*}{ No. of PMCs analysed } & \multirow{2}{*}{$\begin{array}{l}\text { Chromosome } \\
\text { associations }\end{array}$} & \multicolumn{8}{|c|}{ Observed frequency (\%) per set of three homo(eo)logous chromosomes } \\
\hline & & & 1 & 2 & 3 & 4 & 5 & 6 & 7 & 8 \\
\hline \multirow[t]{2}{*}{$\mathrm{BC}_{1}-1$} & 57 & III & $94.7^{*}$ & $80.7^{*}$ & $84.2^{*}$ & 7.0 & $93.0^{*}$ & 3.5 & 15.8 & $82.5^{*}$ \\
\hline & & $\mathrm{II}+\mathrm{I}$ & 5.3 & 19.3 & 15.8 & 93.0 & 7.0 & 96.5 & 84.2 & 17.5 \\
\hline \multirow[t]{2}{*}{$\mathrm{BC}_{1}-2$} & 294 & III & $100^{*}$ & 6.1 & $3.0^{\mathrm{a}}$ & $3.0^{\mathrm{a}}$ & $100^{*}$ & 4.8 & 5.4 & 4.1 \\
\hline & & $\mathrm{II}+\mathrm{I}$ & 0 & 93.9 & 97.0 & 97.0 & 0 & 95.2 & 94.6 & 95.9 \\
\hline \multirow[t]{2}{*}{$\mathrm{BC}_{1}-3$} & 134 & III & 30.6 & $91.0^{*}$ & $97.0^{*}$ & $93.3^{*}$ & $81.3^{*}$ & $40.3^{*}$ & 10.4 & 11.2 \\
\hline & & II+I & 69.4 & 9.0 & 3.0 & 6.7 & 18.7 & 59.7 & 89.6 & 88.8 \\
\hline \multirow{2}{*}{$\mathrm{BC}_{1}-4$} & 57 & III & 57.9 & 12.3 & 14.0 & $84.2^{*}$ & $98.2^{*}$ & 10.5 & $75.4^{*}$ & 14.0 \\
\hline & & $\mathrm{II}+\mathrm{I}$ & 42.1 & 87.7 & 86.0 & 15.8 & 1.8 & 89.5 & 24.6 & 86.0 \\
\hline
\end{tabular}

aSets 3 and 4 could not be distinguished in all MI cells. Data of these sets were added resulting in identical trivalent frequencies.

*Indicates sets containing recombinant chromosomes (see Figure 1 for the sizes and locations of exchanged segments).

tions at metaphase I of this chromosome with its homo(eo)logues. The types of observed chromosome configurations are shown in Figures 2c1-3 and 2d1-3. In the trivalents, the pseudo-iso-chromosome was frequently present as a ring (Figure 2c2, 2d2). At low frequencies $(2.2 \%)$, this chromosome was observed as a ring univalent (Figure 2c3, 2d3). This observation is in agreement with the assumed chromosome morphology.

\section{Meiotic transmission of the $A$. aurea and $A$. inodora chromosomes}

At anaphase I all nonrecombinant $A$. inodora chromosomes, and most of the recombinant $A$. inodora and $A$. aurea chromosomes, segregated regularly to both poles in all $\mathrm{BC} 1$ plants (Figures 2e-i). In contrast, the nonrecombinant $A$. aurea chromosomes and some of the recombinant chromosomes remained in the equatorial plane. At later stages, their chromatids disjoined and migrated to both poles (Figures 2e-i). At late anaphase I/ telophase I, most of the A. aurea chromatids, although still lagging, were equally distributed to both poles in most cells (Figure 2j). In some telophase I cells the metacentric chromatids were divided in the centromeric regions (Figure $2 \mathrm{j}$, chromosome 1A). At anaphase II, the $A$. inodora chromosomes were evenly distributed to both poles, whereas the A. aurea chromosomes were lagging in the equatorial plane and seemed to migrate randomly to both poles. Tetrad stages showed microspores with a different number of A001-I sites, thus confirming the unequal distribution of the A. aurea chromosomes in the gametes. Many of the gametes possessed one to three micronuclei, in which most of the A. aurea chromosomes were present (data not shown).

At anaphase I of the BC1-2 and BC1-3 plants, bridges were frequently observed, especially in the latter (in $75 \%$ of all AI cells). Usually the bridges, which occurred in the nonrecombinant chromosomes 8P in BC1-3 (Figure 2e, f), were accompanied by an acentric fragment (Figure 2h, i), indicating crossovers in a paracentric inversion loop.

The low frequencies of associated homoeologous chromosomes at metaphase I indicated that homoeologous crossover occurs in the $\mathrm{BC} 1$ plants. Evidence for this was observed at anaphase I. Since most of the individual chromosomes could be identified even at anaphase I, it was possible to confirm which chromosomes actually contained novel recombinations (Figure $2 \mathrm{~h}$, i; yellow arrows). Although only few anaphase I cells could be analysed in detail, the occurrence of homoeologous recombination seemed to be similar, as predicted from the homoeologous associations at metaphase I. For example, at anaphase I of the plant $\mathrm{BC} 1-3$, more novel crossovers were observed in the chromosomes 1 than in the smaller chromosomes 7 and 8, which was expected from the pairing behaviour of these chromosomes at metaphase I (Table 1). As a consequence of the crossover and the involvement in trivalent chromosome configurations, the homoeologous recombination allowed these chromosomes to segregate regularly with their homo(eo)logues at anaphase I. This was observed for all chromosomes containing novel homoeologous recombinations.

Figure 2 Fluorescent in situ hybridization applied to metaphase I and anaphase I spreads of BC1-3. GISH was applied to differentiate both parental genomes, A. aurea (red) and A. inodora (green), and sequential FISH with D32-13 (red) and A001-I (green) allowed identification of all individual chromosomes. DAPI (blue) was used as a counterstain. The blue arrows indicate A. aurea chromosomes or chromatids. (a,b) GISH and sequential FISH with D32-13 and A001-I allowed identification of all chromosomes involved in the chromosome configurations. Note that the recombinant chromosome $6 \mathrm{~A} / 6 \mathrm{P}$ is present as a univalent. Homoeologous pairing was observed in the trivalent of non-recombinant chromosomes 1. (c1-3) Different chromosome configurations for the homo(eo)logous chromosomes $2 \mathrm{~A} / 2 \mathrm{P}, 2 \mathrm{P} / 2 \mathrm{~A}$ and $2 \mathrm{P}$ prove the presence of two homologous segments on both arms of $2 \mathrm{~A} / 2 \mathrm{P}$ (see text). (d1-3) Schematic drawings of the chromosome configurations shown in (c1-3). Red and yellow indicate A. aurea and A. inodora chromatin, respectively, and purple indicates the D32-13 hybridization sites. (d4) Ideograms of the different chromosomes 2 present in BC1-3. Note the pseudo-iso-chromosome 2A/2P. (e,f) GISH and FISH applied sequentially to the same anaphase I. In these stages all chromosomes could be identified, however, due to recombination most chromosomes possess different recombined segments and therefore only the centromeric regions of these chromosomes are identified. The bridge (white arrow) between the long arms of the nonrecombinant chromosomes $8 \mathrm{P}$ was often observed in combination with a small a-centric fragment (white arrows in $(\mathbf{h}, \mathbf{i})$ ). (g) Regular migration of the A. inodora chromosomes to both poles, whereas four chromosomes (blue arrows) remain in the equatorial plane. (h,i) A novel recombination (yellow arrows) between two homoeologous chromosome $1 \mathrm{~A}$ and $1 \mathrm{P}$. Note the a-centric fragment of chromosome $8 \mathrm{P}$ (white arrow) and the lagging $A$. aurea chromosomes. (j) Telophase I. Note segregation of $A$. aurea chromatids and the centromere breakage in one of the chromatids of chromosome 1A. White arrow indicates the small a-centric fragment of $8 \mathrm{P}$. 


\section{Discussion}

Compared to meiotic analysis by GISH alone (cf. Parokonny et al, 1994, 1997; King et al, 1994; Benavente et al, 1996), sequential GISH/FISH analysis allowed identification of parental genomes through GISH, and simultaneous identification of all individual chromosomes in the same cell, based on FISH with repetitive probes. This enabled to study the pairing behaviour of all individual chromosomes in allotriploid Alstroemeria hybrids.

\section{Meiotic chromosome behaviour}

A strong correlation was found between the high percentage of trivalents, in which only homologous chromosome segments were associated, and the occurrence of recombinant chromosomes in the BC1-plants. These recombinant chromosomes originated during meiosis in the diploid $A$. aurea $\times A$. inodora hybrid, where high frequencies of homoeologous chromosome pairing ( $80 \%$ heteromorphic bivalents) and recombination were observed (Kamstra et al, 1999a, b). Apparently, the genomes of $A$. aurea and $A$. inodora, although distinguishable in GISH which suggests substantial (repetitive) DNA divergence, remain related to such an extent that homoeologous pairing does occur when homologous chromosomes are absent. A similar situation was observed for the diploid interspecific hybrid $(2 n=2 x=16)$ between Astroemeria ligtu L. $(2 n=2 x=16)$ and $A$. pelegrina L. var. rosea $(2 n=2 x=16)$, where partial homoeologous chromosome association resulted in bivalent formation and chances for homoeologous recombination (Ishikawa and Ishizaka, 2002). An amphidiploid interspecific hybrid $(4 x=32)$ from these species mainly produced bivalents of homologous chromosomes.

The degree of homo(eo)logy between the parental genomes in interspecific hybrids seems fundamental for the occurrence of intergenomic recombination in successive backcross progenies. In general, chromosomes of closely related genomes tend to pair more often than chromosomes of genomes that are more distantly related (Kimber and Yen, 1990). Due to low parental genome homoeology in an allotriploid hybrid between $N$. plumbaginifolia and $N$. sylvestris, monosomic addition lines were obtained in $\mathrm{BC} 1$ and $\mathrm{BC} 2$ for most $N$. sylvestris chromosomes upon backcrossing to N. plumbaginifolia, whereas only a few chromosome translocations were observed (Suen et al, 1997). In monosomic Brassica campestris-alboglabra addition lines, obtained from backcrosses between the less distant resynthesized $B$. napus and $B$. campestris, segmental intergenomic homoeology was observed between the parental genomes that could explain the occurrence of intergenomic introgression in the progenies of these addition lines (Chen et al, 1997).

The level of recombination in backcross progenies of interspecific hybrids is often lower than in the original hybrids (Chetelat et al, 2000). Reduced homoeologous pairing and recombination were also observed during meiosis of the allotriploid BC1-plants derived from the $A$. aurea $\times A$. inodora hybrid when compared to the hybrid itself. This could be fully attributed to preferential homologous pairing in the BC1 plants that contain an additional set of $A$. inodora chromosomes. While suppression of homoeologous pairing and recombination may have a stabilizing effect on the genome constitution of backcross plants, it may hamper intergenomic introgression for breeding purposes.

\section{Genomic stability of Alstroemeria allotriploids}

Newly formed (allo)polyploids, often allotetraploids, generally show considerable meiotic complexity, which is exemplified by the frequent occurrence of phenomena such as multivalent pairing and the production of unbalanced gametes (Ramsey and Schemske, 2002). These meiotic aberrations imply that neopolyploid progenies may include high frequencies of aneuploids, pseudoeuploids and genotypes with recombinant homoeologous chromosomes. Restoration of diploid-like meiotic pairing and selection against meiotic configurations that produce unbalanced gametes generally result in the restoration of fertility in successive generations (Ramsey and Schemske, 2002; Comai et al, 2003).

Although these characteristics broadly apply to our findings, the situation for the allotriploid Alstroemeria hybrids is slightly different. They originated through $2 n-$ gamete formation in the interspecific $A$. aurea $\times A$. inodora hybrid that was backcrossed with $A$. inodora (Kamstra et al, 1999a). Homoeologous pairing in the hybrid yielded varying numbers of recombinant chromosomes (Kamstra et al, 1999b), which led to a high frequency of trivalents (Table 1), affecting meiotic stability and fertility in the BC1 plants. Another factor that complicates genomic stability is the fact that the $A$. aurea chromosomes have no counterparts for meiotic pairing. This can be overcome by either a gradual loss of $A$. aurea chromosomes, which are often observed as univalents in meiosis and in micronuclei in gametes of $\mathrm{BC} 1$ plants, or limited intergenomic introgression of $A$. aurea chromosome segments through homoeologous recombination. The latter possibility is supported by the observation of low frequencies of homoeologous association in metaphase I and homoeologous crossovers in meiosis of the BC1 plants. Taken together, it is doubtful whether the BC1 plants will evolve into genomically stable allotriploids. A more likely result is a diploid constitution in which $A$. aurea chromosome segments have been introgressed into homoeologous A. inodora chromosomes.

\section{Implications for introgression of alien chromosomes} Homoeologous chromosome pairing which may result in introgression was observed at low frequencies in all BC1 plants, with variation among plants and individual chromosomes. In particular, the nonrecombinant $A$. aurea chromosome 1 in BC1-3 and BC1-4 was frequently associated with the homoeologous chromosome 1 from $A$. inodora (Table 1). In $A$. aurea and $A$. inodora, chromosome 1 is large compared to other chromosomes (Buitendijk et al, 1997); therefore chromosome size seems to influence the pairing frequency, as was shown in wheat (Bernardo et al, 1988; Cuadrado et al, 1997).

The random segregation of nonrecombinant $A$. aurea chromosomes at anaphase II and telophase II suggested that $\mathrm{BC} 2$ plants may contain random numbers of $A$. aurea chromosomes. Theoretically, monosomic addition lines could be obtained in BC2, as was found for other plant species (Peffley et al, 1985; Morgan, 1991; Mesbah et al, 1996; Shigyo et al, 1996; Chen et al, 1997; Suen et al, 1997). Many gametes of the BC1 plants contained one to three 
micronuclei with $A$. aurea chromosomes. The question remains whether these micronuclei might hamper fertility and whether the chromosomes in the micronuclei can be transmitted to the progeny. The amount of stainable pollen per BC1 plant varied from 30 to $60 \%$ (Kamstra et al, 1999a). Despite this, only a few BC2 plants were obtained after extensive backcrossing, indicating that additional factors may reduce the actual viability of gametes of the $\mathrm{BC} 1$ plants. Cytogenetic analysis of $\mathrm{BC} 2$ plants could reveal the genomic constitution of successful gametes.

\section{References}

Benavente E, Fernández-Calvín B, Orellana J (1996). Relationship between the levels of wheat-rye metaphase I chromosomal pairing and recombination revealed by GISH. Chromosoma 105: 92-96.

Bernardo A, Diaz F, Jouve N (1988). Chromosome factors affecting pairing in progenies of $6 x$-triticale $\times$ Triticum turgidum L spp. turgidum conv. durum (Desf.). Heredity 60: 455-461.

Buitendijk JH, Boon EJ, Ramanna MS (1997). Nuclear DNA content in twelve species of Alstroemeria L and some of their hybrids. Ann Bot 79: 343-353.

Chen BY, Cheng BF, Jørgensen RB, Heneen WK (1997). Production and cytogenetics of Brassica campestris-alboglabra chromosome addition lines. Theor Appl Genet 94: 633-640.

Chetelat RT, Meglic V, Cisneros P (2000). A genetic map of tomato based on BC1 Lycopersicon esculentum $\times$ Solanum lycopersicoides reveals overall synteny but suppressed recombination between these homoeologous genomes. Genetics 154: 857-867.

Comai L, Tyagi AP, Lysak MA (2003). FISH analysis of meiosis in Arabidopsis allopolyploids. Chrom Res 11: 217-226.

Cuadrado A, Vitellozzi F, Jouve N, Ceoloni C (1997). Fluorescence in situ hybridization applied to the analysis of wheatrye chromosome pairing. Theor Appl Genet 94: 347-355.

Goemans JAM (1962). Breeding of Alstroemerias. J R Hortic Soc 87: 282-284.

Ishikawa T, Ishizaka H (2002). Chromosome association and Giemsa C-banding of meiotic chromosomes in interspecific hybrid of Alstroemeria ligtu L. hybrid and A. pelegrina L. var. rosea, its amphidiploid, and allotriploid between the amphidiploid and the parents. Breed Sci 52: 27-33.

Kamstra SA, Kuipers AGJ, De Jeu MJ, Ramanna MS, Jacobsen E (1997). Physical localization of repetitive DNA sequences in Alstroemeria: karyotyping of two species with species-specific and ribosomal DNA. Genome 40: 652-658.

Kamstra SA, Ramanna MS, De Jeu MJ, Kuipers AGJ, Jacobsen E (1999a). Homoeologous chromosome pairing in the distant Alstroemeria aurea $\times A$. inodora and the genome composition of its backcross derivatives determined through fluorescent in situ hybridization with species-specific probes. Heredity 82: 69-78.
Kamstra SA, Ramanna MS, De Jeu MJ, Kuipers AGJ, Jacobsen E (1999b). The extent and position of homoeologous recombination in a distant hybrid of Alstroemeria: a molecular cytogenetic assessment of first generation backcross progenies. Chromosoma 108: 52-63.

Kimber G, Yen Y (1990). Genome analysis of diploid plants. Proc Natl Acad Sci USA 87: 3205-3209.

King IP, Reader SM, Purdie KA, Orford SE, Miller TE (1994). A study of the effect of a homoeologous pairing promotor on chromosome pairing in wheat/rye hybrids using genomic in situ hybridization. Heredity 72: 318-321.

Kuipers GJ, van Os DPM, de Jong JH, Ramanna MS (1997). Molecular cytogenetics of Alstroemeria: identification of parental genomes in interspecific hybrids and characterization of repetitive DNA families in constitutive heterochromatin. Chrom Res 5: 31-39.

Mesbah M, De Bock TSM, Sandbrink JM, Klein-Lankhorst RM, Lange W (1996). Selection of monosomic addition plants in offspring families using repetitive DNA probes in Beta L. Theor Appl Genet 92: 891-897.

Morgan WF (1991). The morphology and cytology of monosomic addition lines combining single Festuca drymeja chromosomes and Lolium multiflorum. Euphytica 55: 57-64.

Parokonny AS, Kenton A, Gleba YY, Bennett MD (1994). The fate of recombinant chromosomes and genome interaction in Nicotiana asymmetric somatic hybrids and their sexual progeny. Theor Appl Genet 89: 488-497.

Parokonny AS, Marshall JA, Bennett MD, Cocking EC, Davey MR, Power JB (1997). Homeologous pairing and recombination in backcross derivatives of tomato somatic hybrids (Lycopersicon esculentum (+) L. peruvianum). Theor Appl Genet 94: 713-723.

Peffley EB, Corgan JN, Horak KE, Tanksley SD (1985). Electrophoretic analysis of Allium alien addition lines. Theor Appl Genet 71: 176-184.

Ramanna MS (1992). The role of sexual polyploidization in the origin of horticultural crops: Alstroemeria as an example. In: Mariani A, Tavoletti S (eds) Proceedings of the Workshop: Gametes with Somatic Chromosome Number in the Evolution and Breeding of Polyploid Polysomic Species: Achievements And Perspectives. Tipolitografia Porziuncola Assisi: Perugia, Italy. pp 83-89.

Ramsey J, Schemske DW (2002). Neopolyploidy in flowering plants. Annu Rev Ecol Syst 33: 589-639.

Shigyo M, Tashiro Y, Shiro I, Miyazaki S (1996). Establishment of a series of alien monosomic addition lines of Japanese bunching onion (Allium fistulosum L.) with extra chromosomes from shallot (A. cepa L. Aggregatum group). Genes Genet Syst 71: 363-371.

Suen DF, Wang CK, Lin RF, Kao YY, Lee FM, Chen CC (1997). Assignment of DNA markers to Nicotiana sylvestris chromosomes using monosomic alien addition lines. Theor Appl Genet 94: 331-337. 